\title{
INTERGALACTIC GAS
}

\author{
GEORGE B. FIELD \\ Center for Astrophysics, Harvard College Observatory \\ and \\ Smithsonian Astrophysical Observatory, Cambridge, Mass., U.S.A.
}

\begin{abstract}
Evidence that rich clusters of galaxies contain hot $\left(T=10^{8} \mathrm{~K}\right)$ intracluster gas is reviewed. Such gas contributes little to $\Omega(0.003)$ but it has been argued that $\Omega$ must be less than 0.05 for true intergalactic gas, if accretion of more gas than is observed in rich clusters is to be avoided. This argument is reviewed.

If the de Vaucouleur' groups are bound by intracluster gas, $T$ is expected to be $10^{5}$ to $10^{7} \mathrm{~K}$ and the contribution to $\Omega$ is $\simeq 1$. Since the clumping factor $C$ is estimated to be $\simeq 7$, the resulting value of $\Omega^{2} C$ is $\simeq 7$. This does not violate the observed diffuse soft X-ray background intensity. Gas should be sought in such groups. Smoothly distributed gas with $10^{7}<T<3 \times 10^{8} \mathrm{~K}$ and $\Omega=1$ is not ruled out by direct observations.
\end{abstract}

\section{Introduction}

Since a recent review (Field, 1972) was written, positive indications of intergalactic gas (IGG) have been found. I shall skip rapidly over the negative results found earlier in order to concentrate on these positive indications and their interpretation in terms of the cosmological parameter $\Omega \equiv \varrho / \varrho_{c}\left(\varrho_{c}=3 H^{2} / 8 \pi G\right)$.

How one interprets the data from various observations depends upon the degree to which IGG is clumped, as indicated by the parameter $C=\left\langle\varrho^{2}\right\rangle\left\langle\underline{\varrho^{2}}\right\rangle^{2}$. At present, the value of $C$ can only be guessed at; Silk and Tarter (1973) make the point that since $C \gg 1$ for luminous matter in the Universe, one might expect the same to be true for IGG.

In Section 2, I discuss the (unlikely) case that $C=1$ and show that while neutral gas contributes $\Omega \ll 1$, ionized gas could in principle contribute $\Omega \simeq 1$ if the diffuse X-ray background is interpreted as thermal bremsstrahlung (TB).

In Section 3, I review the recent X-ray evidence that rich clusters of galaxies contain hot intracluster gas (ICG). Although such gas per se contributes $\Omega \ll 1$, theory suggests that.ICG is connected with IGG. Hence one ultimately may be able to deduce something about IGG from ICG. Some theory indicates that ICG in small groups of galaxies may contribute significantly to $\Omega$. Section 4 is a summary.

\section{Smoothly Distributed Gas}

If $C=1$, the predicted observational properties of IGG depend upon $\Omega, T$ (the temperature), and $x$ (the ionization fraction). First consider the case $T<10^{4} \mathrm{~K}$, in which case $x \ll 1$. Then IGG is either $\mathrm{HI}$ or $\mathrm{H}_{2}$. Lack of $\mathrm{L} \alpha$ and Lyman-band absorption in quasars with redshift $z \simeq 2$ implies that $\Omega(\mathrm{HI})<3 \times 10^{-7}$ and $\Omega\left(\mathrm{H}_{2}\right)<7 \times 10^{-5}$ at that redshift (Field, 1972). The Lyman-band observations also rule out intergalactic dust grains of solid $\mathrm{H}_{2}$ (Purcell, 1973). 
HI has been sought directly at low $z$ by $21-\mathrm{cm}$ techniques with the result that $\Omega<0.16$ (Field, 1972). A much stronger limit follows from theory applied to the observations at $z=2$. If the $z=2$ gas is ionized by collisions, $T(z=2)$ must exceed $2 \times 10^{6} \mathrm{~K}$ for $\Omega(\mathrm{IGG}) \simeq 1$. Even the most rapid cooling would give $T(z=0)>3 \times 10^{5} \mathrm{~K}$ and hence $y \equiv 1-x<10^{-4}$. Alternatively, a model based on photoionization with $T \sim 10^{4} \mathrm{~K}$ (Arons and Wingert, 1972), which satisfies the observations at $z=2$, yields $y<10^{-5}$ at $z=0$. I conclude that $\Omega(\mathrm{HI})<10^{-4}$ at $z=0$.

In analogy with interstellar gas (ISG), one may consider the presence of intergalactic dust grains, particularly if the gas is not too hot. Earlier work showed that if intergalactic dust is like interstellar dust and has the same ratio to gas as in the Galaxy, then $\Omega<0.06$ (Field, 1972). McKee and Petrosian (1974) have sharpened this limit by searching for the $2200 \AA$ feature, which is prominent in interstellar extinction, in the spectra of quasars of large redshift. Their upper limit corresponds to $\Omega<0.004$. Neither result is very helpful if, as expected, the dust-to-gas ratio is smaller in intergalactic space because of the low abundance of heavy elements there.

If $T>10^{4} \mathrm{~K}$, the gas is ionized, and other observational methods are appropriate. Since heavy elements may be underabundant, the best methods are those sensitive to a gas containing only free electrons, together with $\mathrm{H}$ and $\mathrm{He}$ ions. For $T>10^{6} \mathrm{~K}$, one such method is observation of TB in the X-ray region. The observed diffuse X-ray background may be due to this mechanism. A simple integration along the light cone back to some maximum redshift $z_{\max }$ yields a theoretical spectrum (Field and Henry, 1964). In Figure $1 \mathrm{I}$ show the spectrum for $T_{0} \equiv T(z=0)=2 \times 10^{8} \mathrm{~K}$ and $z_{\max }=1$. It was assumed that $T$ decreases adiabatically with $\gamma=\frac{5}{3}$ from $z=1$ to $z=0$, that $H_{0}=$ $50 \mathrm{~km} \mathrm{~s}^{-1} \mathrm{Mpc}^{-1}$, and that $\Omega(\mathrm{IGG})=1$. The Gaunt factor was taken to be unity (which is correct within a factor of 2). Since the theoretical intensities in this case only barely exceed those observed, one cannot conclude that $\Omega<1$ for such a gas. The same is true for all temperatures down to $10^{7} \mathrm{~K}$ (solid line of slope $-\frac{1}{2}$ ) and since the observations below $1 \mathrm{keV}$ actually exceed the theoretical value, $\Omega$ could actually exceed 1 for $T \sim 10^{6} \mathrm{~K}$. However, $\Omega<1$ for $T>3 \times 10^{8} \mathrm{~K}$, since otherwise the observations would rise up to the solid line somewhere above $100 \mathrm{keV}$. In summary, $\Omega=1$ is not ruled out for IGG with $T=10^{6}-3 \times 10^{8} \mathrm{~K}$.

What if $10^{4}<T<10^{6} \mathrm{~K}$ ? Here the TB emission would lie between $1 \mathrm{eV}$ and $100 \mathrm{eV}$, in a region which is obscured at the low end by starlight and at the high end by interstellar absorption. While TB is augmented by line radiation excited by recombination and by collisions in this temperature range, the redshift would spread these into a continuum not very much stronger than TB. Hence there is a paucity of observational limits on IGG with $T=10^{4}-10^{6} \mathrm{~K}$.

\section{Intracluster Gas}

In the past few years several rich clusters of galaxies have been found to be X-ray sources. The emission mechanism is uncertain, TB and inverse compton (IC) being prime contenders. If it is TB, one infers that a mass of gas equal to a few percent of the 


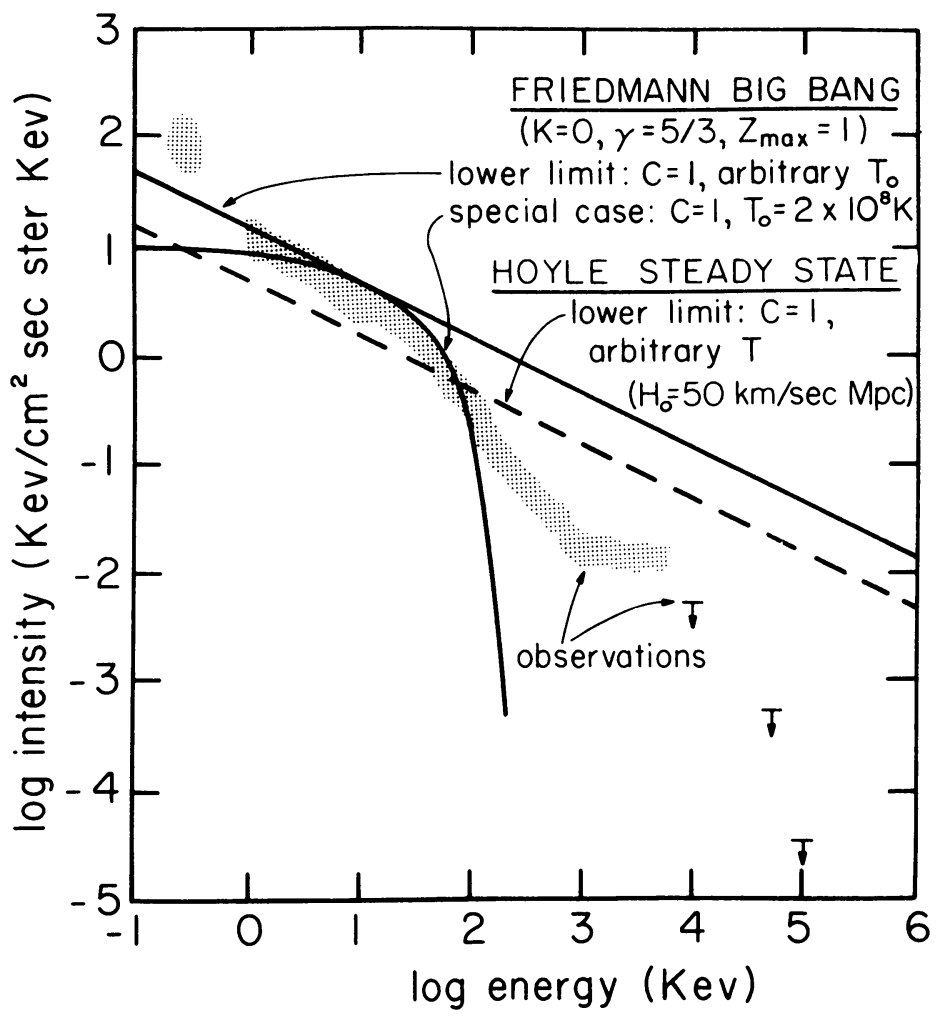

Fig. 1. The observed X-ray background (shaded 'leg') superposed on theoretical TB spectra for the Universe, integrated back to $z=1$ (Field, 1972). The special case corresponds to $T_{0} \equiv T(z=0)=2 \times 10^{8} \mathrm{~K}$, rising adiabatically with $\gamma=\frac{5}{3}$ as $z$ increases. It is assumed that $\Omega=C=1$. The solid line with slope $-\frac{1}{2}$ is the envelope of curves of various $T_{0}$. If $\Omega=1$, the observations must reach up to this limiting curve at some point, and above it if $C>1$. Thus, one concludes that $T_{0}<3 \times 10^{8} \mathrm{~K}$ if $\Omega=1$, but $\Omega=1$ is not ruled out for $10^{6}<T_{0}<3 \times 10^{8} \mathrm{~K}$. Some authors have suggested that the $30-\mathrm{keV}$ 'knee' can be explained by a dominant component at $2 \times 10^{8} \mathrm{~K}$.

virial mass of the cluster may be present, with $T \simeq 10^{8} \mathrm{~K}$. If this interpretation is correct, the $\mathrm{X}$-ray observations are the first to give positive indications of gas outside of galaxies.

The first cluster showing the effect was Coma (Meekins et al., 1971; Gursky et al., 1971). The Third UHURU Catalog (Kellogg et al., 1973; Gursky, 1973) has yielded 9 definite and 8 possible identifications with Abell clusters, including Perseus, Coma, and Hercules, and 4 with other clusters, including Virgo. Of the 13 Richness-II clusters given by Abell within distance class 3, 6 are X-ray sources, indicating that X-ray emission in the 2-8 keV band of UHURU is a common property of rich clusters.

The most careful study has been made of Coma, which, like 5 other cluster sources, has a finite angular size. Lea et al. (1973) fitted various density distributions to the $\mathrm{X}$-ray observations of the Coma cluster, including (1), $\left(1+r^{2} / a^{2}\right)^{-1}$ and (2), $(1+$ $\left.+r^{2} / a^{2}\right)^{-3 / 2}$. They assumed TB at a constant $T=9 \mathrm{keV}=1.0 \times 10^{8} \mathrm{~K}$ (see below) and 
took $C=1$. The results for case (2) are shown in Figure 2, and model parameters for both cases are given in Table I.

TABLE I

TB models of the Coma Cluster

\begin{tabular}{lllll}
\hline Model & $\begin{array}{l}\text { Assumed } \\
\text { temperature } \\
(\mathrm{K})\end{array}$ & $\begin{array}{l}\text { Core } \\
\text { radius, } a \\
(\operatorname{arcmin})\end{array}$ & $\begin{array}{l}\text { Central } \\
\text { density } \\
\left(\mathrm{cm}^{-3}\right)\end{array}$ & $\begin{array}{l}\text { Mass of } \\
\text { gas } \\
\left(M_{\odot}\right)\end{array}$ \\
\hline 1 & $1.0 \times 10^{8}$ & 7 & $4 \times 10^{-3}$ & $2 \times 10^{14}$ \\
2 & $1.0 \times 10^{8}$ & 16 & $3 \times 10^{-3}$ & $5 \times 10^{14}$ \\
\hline
\end{tabular}

Both models fit the data equally well. Model 1 is very like the distribution of galaxies. Since the virial mass of Coma is $6 \times 10^{15} M_{\odot}$ and the mass of galaxies is $8 \times 10^{14} M_{\odot}$ (Rood et al., 1972), the mass of gas is $3-8 \%$ of the virial mass, and $25-63 \%$ of the galaxy mass. As these data are based upon $C=1$, they are upper limits; clumping would yield smaller gas masses.

What reason is there to assume $T=1.0 \times 10^{8} \mathrm{~K}$ ? Figure 3 compares the results of a soft X-ray (0.15-2.0 keV) observation of Coma (Gorenstein et al., 1973) with two models of the emission which fit the $2-8 \mathrm{keV}$ photon spectrum of UHURU equally well. The first, a power law $E^{-2}$, is characteristic of IC. The second, $G E^{-1} \exp (-E / k T)$, is characteristic of TB, where $G$ is the Gaunt factor, which depends slightly on $E$. As can be seen, the TB model fits the data much better, with $k T=8.1 \mathrm{keV}$ or $T=0.9 \times$ $\times 10^{8} \mathrm{~K}$.

The one-dimensional rms velocity of a particle, $(R T / \mu)^{1 / 2}$, in a fully ionized gas with $\mathrm{He} / \mathrm{H}=0.1$ is $1100 \mathrm{~km} \mathrm{~s}^{-1}$ at $T=0.9 \times 10^{8} \mathrm{~K}$. This compares with the observed line-of-sight velocity dispersion of the galaxies in the core of Coma, $1060 \mathrm{~km} \mathrm{~s}^{-1}$. Hence it is consistent that the distribution of gas is like that of the galaxies, which are distributed like model (1) with $a=6$ 6 4 (King, 1972).

In summary, the X-ray data on Coma are consistent with TB from ICG having $T=0.9 \times 10^{8} \mathrm{~K}$ and a mass of $(2-5) \times 10^{14} M_{0}$. The fact that many other clusters are $\mathrm{X}$-ray sources is consistent with the assumption that they too contain substantial amounts of ICG.

Other evidence can be cited:

(i) Coma also exhibits a diffuse optical (4000-5000 $\AA$ ) emission which may be due to a cooler component of ICG (Welch and Sastry, 1971, 1972a).

(ii) Coma and other clusters exhibit an unusual effect in which the lobes of radio galaxies within the cluster are distorted as if the magnetic field of the Galaxy were being swept back by the ICG through which the galaxies move (Miley et al., 1972). Figure 4 shows the effect for NGC 1265 in the Perseus cluster (Wellington et al., 1973). NGC 4869 in Coma exhibits a similar effect (Willson, 1970).

(iii) De Young (1972) compared the separations of components of radio galaxies within and outside of clusters, and found that those within are typically only half of 

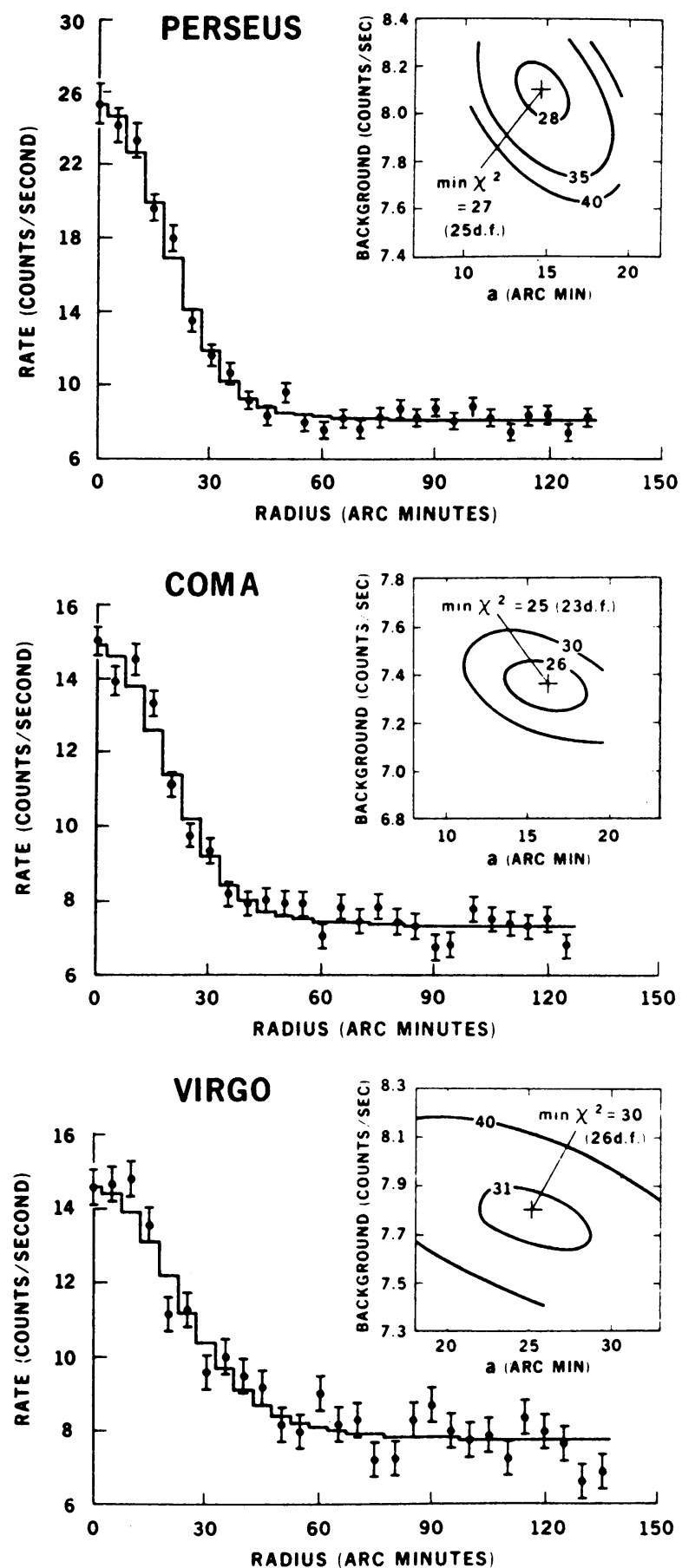

Fig. 2. Comparison between TB model of cluster X-ray sources and UHURU data for 3 clusters. Density is assumed to be distributed like $\left(1+r^{2} / a^{2}\right)^{-3 / 2}$. The $16^{\prime}$ for Coma corresponds to $0.64 \mathrm{Mpc}$ if $H_{0}=50$ (Lea et al., 1973). 


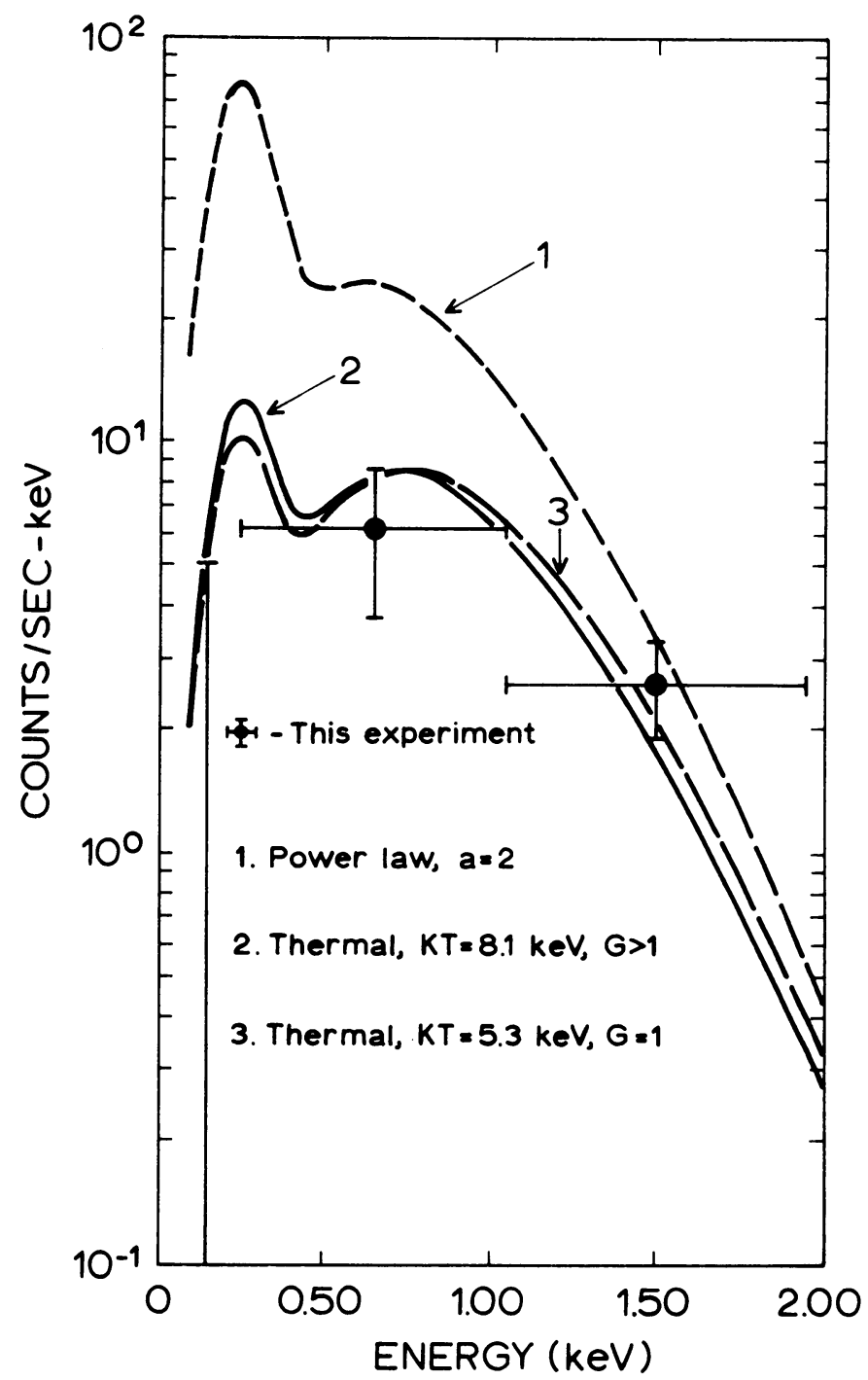

Fig. 3. Comparison between soft X-ray data on Coma and two different models which fit the 2-8-keV UHURU data equally well. The power law is excluded and a good fit is obtained with $k T=8.1 \mathrm{keV}$ if the Gaunt factor $G$ is taken into account (Gorenstein et al., 1973).

those outside (Figure 5). If ram pressure is responsible for confining the components, the density of ICG is about 15 times that of IGG in general.

(iv) Although not much is known in detail about the cluster X-ray sources, there appears to be a correlation between $\mathrm{X}$-ray luminosity, $L_{\mathrm{X}}$, and the velocity spread $\Delta V$ of the cluster galaxies (Figure 6). Solinger and Tucker (1972) argue that if the mass of gas in a cluster is proportional to its virial mass, then origin of the X-rays by TB emission implies that $L_{\mathrm{X}} \propto n^{2} R^{3} \propto M^{2} / R^{3}$. Since from the virial theorem $M \propto R(\Delta V)^{2}$, 


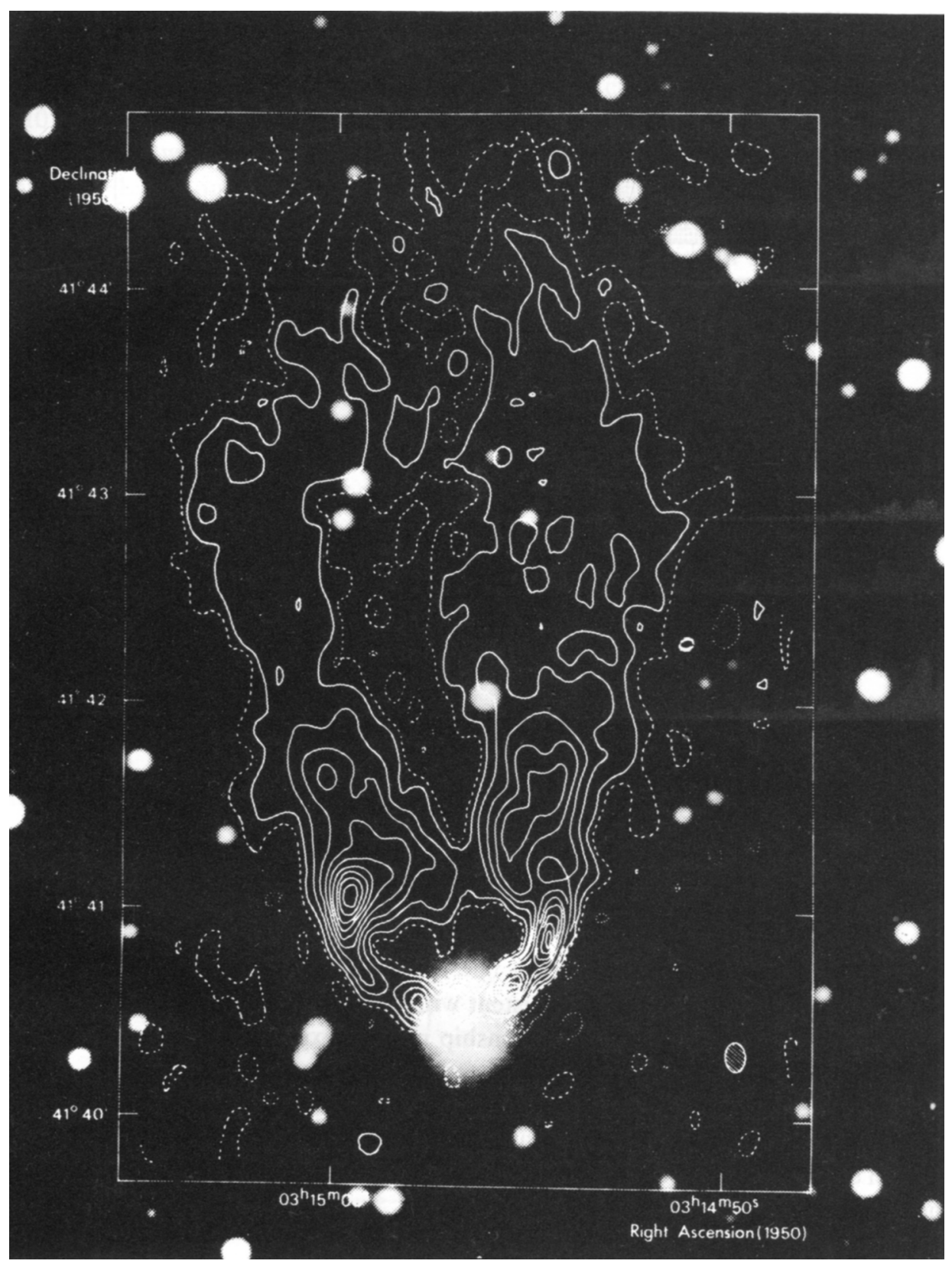

Fig. 4. A Westerbork 5-GHz map of NGC 1265 in the Perseus cluster, superposed on the Palomar Sky Survey blue print. The symmetry of the radio 'tail' is apparent. (Wellington et al.. 1973.) 


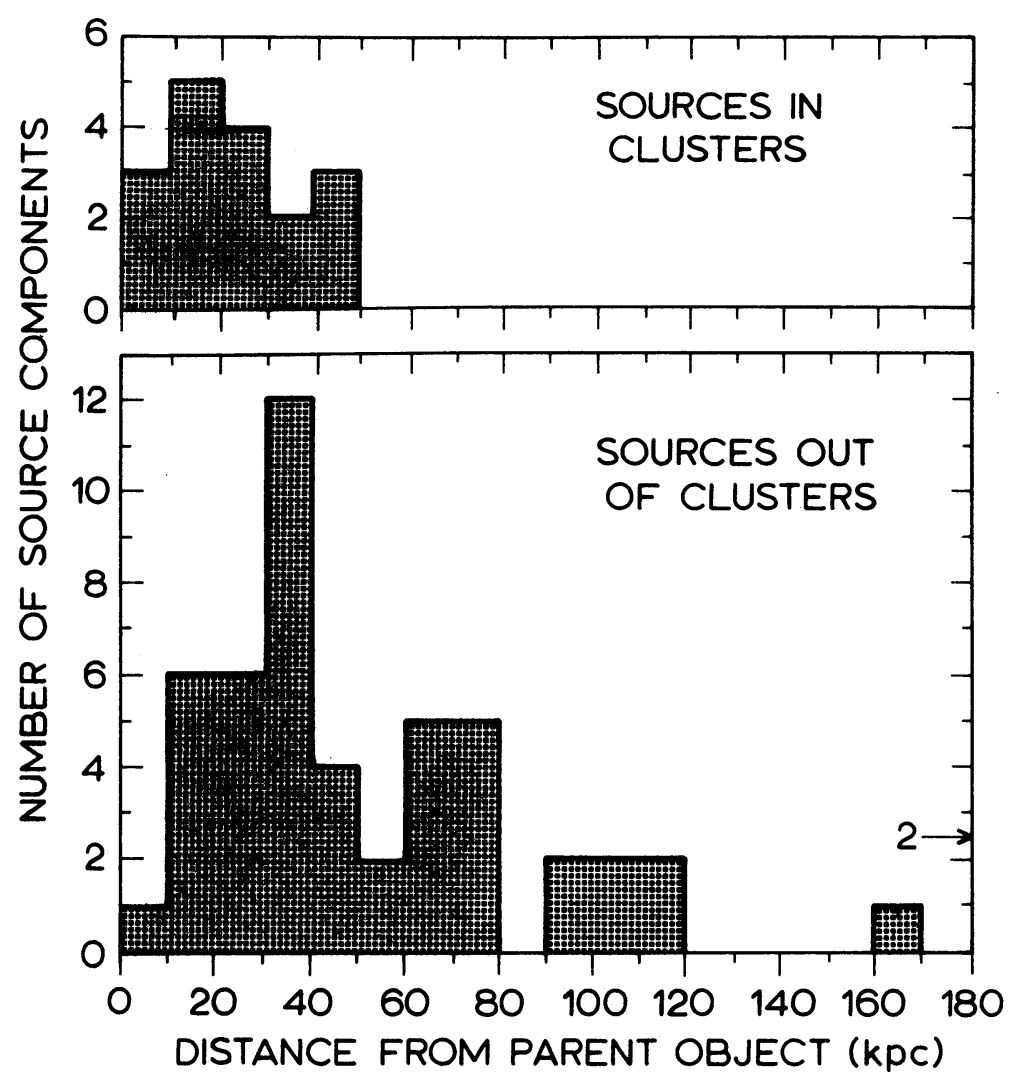

Fig. 5. Distribution of component separations for radio galaxies within (top) and outside of (bottom) clusters. Two sources outside of clusters are off the lower diagram, at 210 and $230 \mathrm{kpc}$. The average separation of those outside of clusters is about double those within (De Young, 1972).

$L_{\mathbf{X}} \propto(\Delta V)^{4} / R$. If the dependence on $R$ is ignored, $L_{\mathrm{X}}$ should be proportional to $(\Delta V)^{4}$, and indeed, Figure 6 is roughly consistent with that relationship. Brown (1973) has argued, however, that the same relationship may be explained on the basis of IC.

(v) There is ambiguous evidence concerning the scattering of the $3 \mathrm{~K}$ cosmic blackbody background radiation by the free electron component of the ICG in Coma. This effect, predicted by Zel'dovich and Sunyaev (1969) and discussed by Sunyaev and Zel'dovich (1972), results in a fractional decrease in brightness temperature toward the cluster of

$$
\frac{\Delta T_{B}}{T_{B}}=-2 \frac{k T}{m c^{2}} \tau,
$$

where

$$
\tau=\int n_{e} \sigma \mathrm{d} l
$$


is the Thompson-scattering optical depth through the cluster. From the X-ray data, one predicts $\Delta T_{B} / T_{B}=-2.5 \times 10^{-4}$, corresponding to $\Delta T_{B}=-8 \times 10^{-4} \mathrm{~K}$. Such an effect is extremely difficult to observe. Pariiskii (1972) claimed to have found $\Delta T \simeq-$ $-10 \times 10^{-4} \mathrm{~K}$ at $\lambda=4 \mathrm{~cm}$ but no details were given. In particular, the correction for beam dilution was not discussed. Davidsen et al. (1973) performed a similar observation at $\lambda=1.3 \mathrm{~cm}$ and obtained a $2 \sigma$ range on their antenna temperature of $\Delta T_{A}=-$ -20 to $+32 \times 10^{-4} \mathrm{~K}$, compared to $\Delta T_{A}=-8 \times 10^{-4} \mathrm{~K}$ expected from the $\mathrm{X}$-ray data. The data of Davidsen et al. have been used below to set an upper limit on the thermal bremsstrahlung radio emission by the gas in Coma.

Both the galaxies and the hot ICG in Coma fail to bind it by about an order of

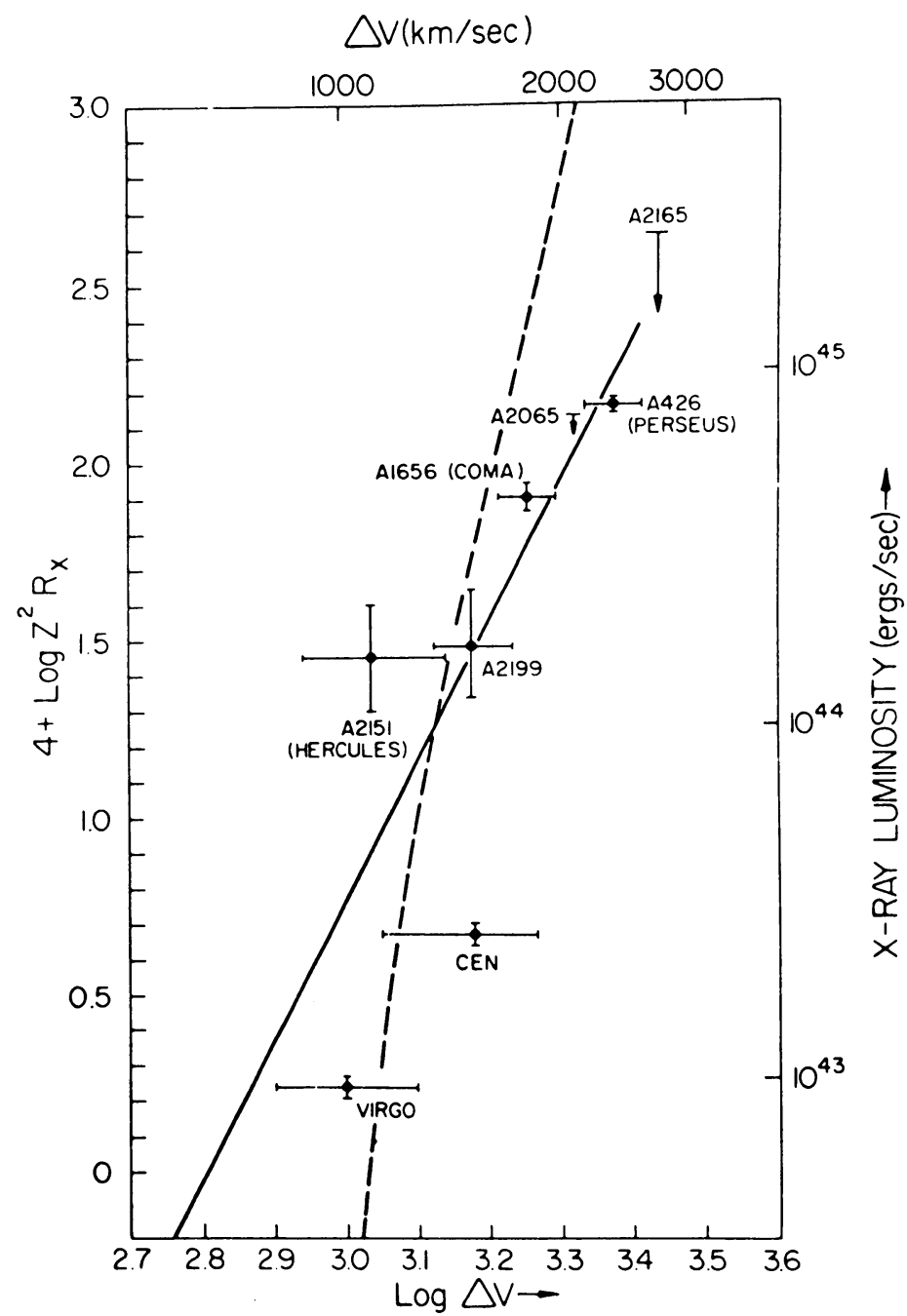

Fig. 6. Relation between X-ray luminosity and velocity spread for clusters of galaxies (Kellogg et al., 1973). The solid line is $L_{\mathrm{X}}=\operatorname{const}(\Delta V)^{4}$. 
magnitude. Interest therefore centers on whether cooler gas may be present. Cooler gas may be sought by $21-\mathrm{cm}$, radio, continuum optical, $L \alpha$, and soft $\mathrm{X}$-ray emission. All these types of observation have been made of Coma, with negative results for $21-\mathrm{cm}$, L $\alpha$, and soft X-ray emission. De Young and Roberts (1974) use 21-cm observations to show that there are less than $1.4 \times 10^{11} M_{\odot}$ of optically thin HI in Coma, a completely negligible amount. They also rule out a model in which the HI is gathered into optically thick clouds (Smart, 1973), so the only alternative is ionized gas of unknown temperature. In Figure 7 the upper limits on $\omega^{2} C$ are plotted, where $C$ is the clumping factor and $\omega=\varrho / \varrho_{c}$, with $\varrho_{c}$ the density needed to bind the cluster $(0.12$ proton masses $\mathrm{cm}^{-3}$ at $r=0$ ). This graph is after that given by Holberg et al.(1973), who carried out the $\mathrm{L} \alpha$ measurement and collected the other data. Since TB is proportional to $\varrho^{2}$, one needs the value of $\int \varrho_{c}^{2} \mathrm{~d} V$ integrated over the cluster. Here the calculations of King (1972), based upon the assumption that the gas is distributed like the galaxies, have been used. This is true for the missing matter (whatever its nature) according to the dynamical study of Rood et al.(1972), and it appears also to be true for the $T=10^{8} \mathrm{~K}$ gas, according to the $\mathrm{X}$-ray observations (see above). Radio and optical emission have been detected from Coma. However, as they could be caused by something else (e.g. synchrotron radiation and starlight), the corresponding values of $\omega^{2} C$ are plotted as upper limits.

From the radio, $L \alpha$, and soft $X$-ray observations alone, one concludes that if $\omega=1$, then $7 \times 10^{4}<T<3 \times 10^{5} \mathrm{~K}$ and $C<2.5$. Since the temperature is low, such gas can be supported against gravity only if it is clumped into clouds which orbit in the cluster. This situation has been studied by Goldsmith and Silk (1972), who conclude that $C$ must be at least 15 in order to avoid various difficulties like cloud-cloud collisions. Hence it appears unlikely that Coma is bound by ionized gas.

One may also utilize the optical observations summarized by Welch and Sastry (1972b) as an upper limit. Even if one includes only the TB from such a gas and neglects the contributions from free-bound and bound-bound transitions, one obtains the curve labelled 'optical' in Figure 7. It happens that the negative result by Davidsen et al. (1973) at $\lambda=1.3 \mathrm{~cm}$, when interpreted in terms of thermal bremsstrahlung, gives a curve very close to that labelled optical in Figure 7. Even if $C=1, \omega^{2}<\frac{1}{4}$ and $\omega<\frac{1}{2}$ from this curve. Since it is likely that $C>1$ and since part of the emission has been neglected, I conclude that it is very unlikely that Coma is bound by ionized gas with $T$ near $10^{5} \mathrm{~K}$. Since other temperatures have been ruled out, I conclude that Coma is not bound by ionized gas of any temperature. Other $L \alpha$ results have been derived for the Perseus and Pegasus I clusters of galaxies by Bohlin et al. (1973).

Even if clusters are not bound by gas, one may possibly learn something from an argument due to Gunn and Gott (1972), who consider the dynamical response of hypothetical IGG to the gravitational attraction of a rich cluster like Coma. There is a critical radius at which the Hubble velocity, $v=H r$, equals the escape velocity from the cluster, $v_{e}=(2 G M / r)^{1 / 2}$, which, for $H=50 \mathrm{~km} \mathrm{~s}^{-1} \mathrm{Mpc}^{-1}$ and $M=6 \times 10^{15} M_{\odot}$, is $27 \mathrm{Mpc}$. Gas inside this radius must fall into the cluster sooner or later. In the process, it may be shock heated to $T_{8} \equiv T / 10^{8} \mathrm{~K} \simeq 1$, sufficient to support it against gravity. 


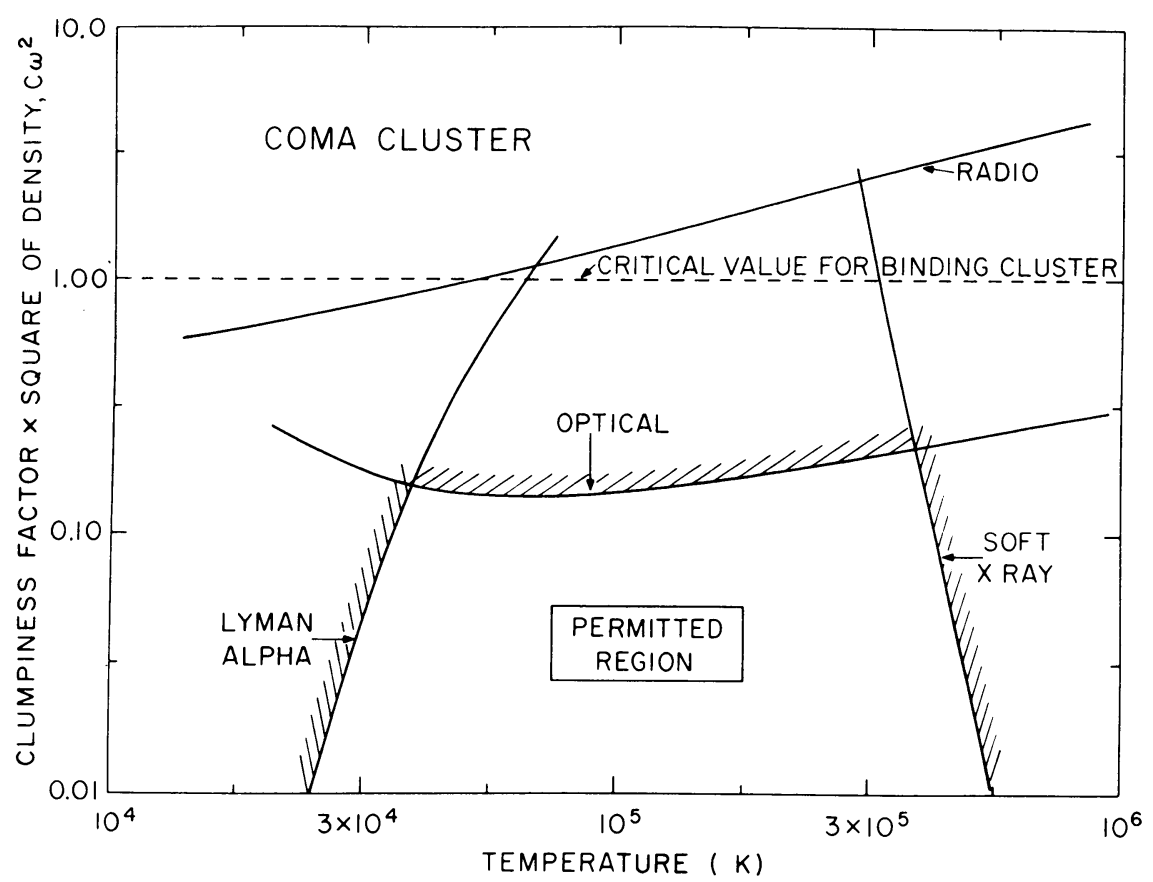

Fig. 7. The values of clumpiness $C$, density $\omega=\varrho / \varrho_{c}$, and temperature $T$ permitted by radio, optical, $L \alpha$, and soft X-ray observations of the Coma cluster. If optical observations are ignored, $7 \times 10^{4}<T<3 \times 10^{5} \mathrm{~K}$ and $\omega^{2} C<2.5$, while if optical observations are taken into account, $\omega^{2} C<\frac{1}{4}$. This result is confirmed by the radio observations of Davidsen et al. (1973). Even if $C=1, \omega<\frac{1}{2}$ (after Holberg et al., 1973).

Gunn and Gott solved the dynamical problem, ignoring all but gravitational forces. As shown in Figure 8, gas within $10 \mathrm{Mpc}$ has already developed a negative radial velocity (infall). The infall has compressed the gas, leading to the density profile of IGG near the cluster shown in Figure 9 for various values of $q_{0} .\left(H_{0}=75\right.$ is assumed here.)

If $\Omega=1\left(q_{0}=\frac{1}{2}\right)$, the present rate of infall is calculated to be $1.5 \times 10^{5} M_{\odot}$ per year, enough to accumulate the 'observed' (X-ray) mass of $6 \times 10^{13} M_{\odot}$ in $4 \times 10^{8} \mathrm{yr}$ (all based on $H_{0}=75$ ). Since this is much less than the age of the Universe, one wonders about the fate of gas which must have fallen in earlier. It cannot have accreted onto the giant galaxies at the center of the cluster, because their observed masses are too small. It cannot be making new galaxies, because none is observed. It cannot have formed cool clouds, because such clouds would give observable emission (see above). The only other possibility is that some repulsive force is preventing gas from falling in. Gunn and Gott consider energetic mass outflow from quasars as a potential candidate but discard it. They therefore conclude that the small amount of accreted gas must mean that $\Omega$ is small, of the order of 0.05 . Since the observed gas could have originated elsewhere, this is an upper limit.

This argument is so important for cosmology that one wonders if there is a way around it. Hydrostatic pressure was ignored by Gunn and Gott, no doubt because 
they believed IGG to be cool. However, there is evidence that IGG is hot, so the argument is worth re-examining. One finds that the density profiles computed by Gunn and Gott (Figure 9) would be accompanied by a significant outward pressure force if $T_{8}=1.5$ at $r=1-3 \mathrm{Mpc}$. The X-ray observations indicate that $T_{8} \simeq 1$ at $\sim 1 \mathrm{Mpc}$, so it is conceivable that $T_{8} \simeq 1$ farther out as well.

One can speculate that the first gas to fall in transferred a considerable amount of energy to the gas outside. If an amount of energy comparable to the present thermal energy in the cluster were transmitted outward, there would have been enough to heat an $\Omega=1$ IGG up to $T_{8}=1$ out to $10 \mathrm{Mpc}$. Crude estimates suggest that thermal conduction might have been able to do this in the time available. Another possibility is that IGG is prevented from falling into Coma by a 'wind' resulting from gas flowing out of galaxies in the cluster. Yahil and Ostriker (1973) have constructed a model in which the X-ray emission is caused by a flow at $10^{3}-10^{4} M_{\odot} \mathrm{yr}^{-1}$ out of the galaxies at $\sim 1000 \mathrm{~km} \mathrm{~s}^{-1}$. This would probably prevent accretion. Until these possibilities are evaluated, Gunn and Gott's conclusion that $\Omega<0.05$ must be treated with some caution.

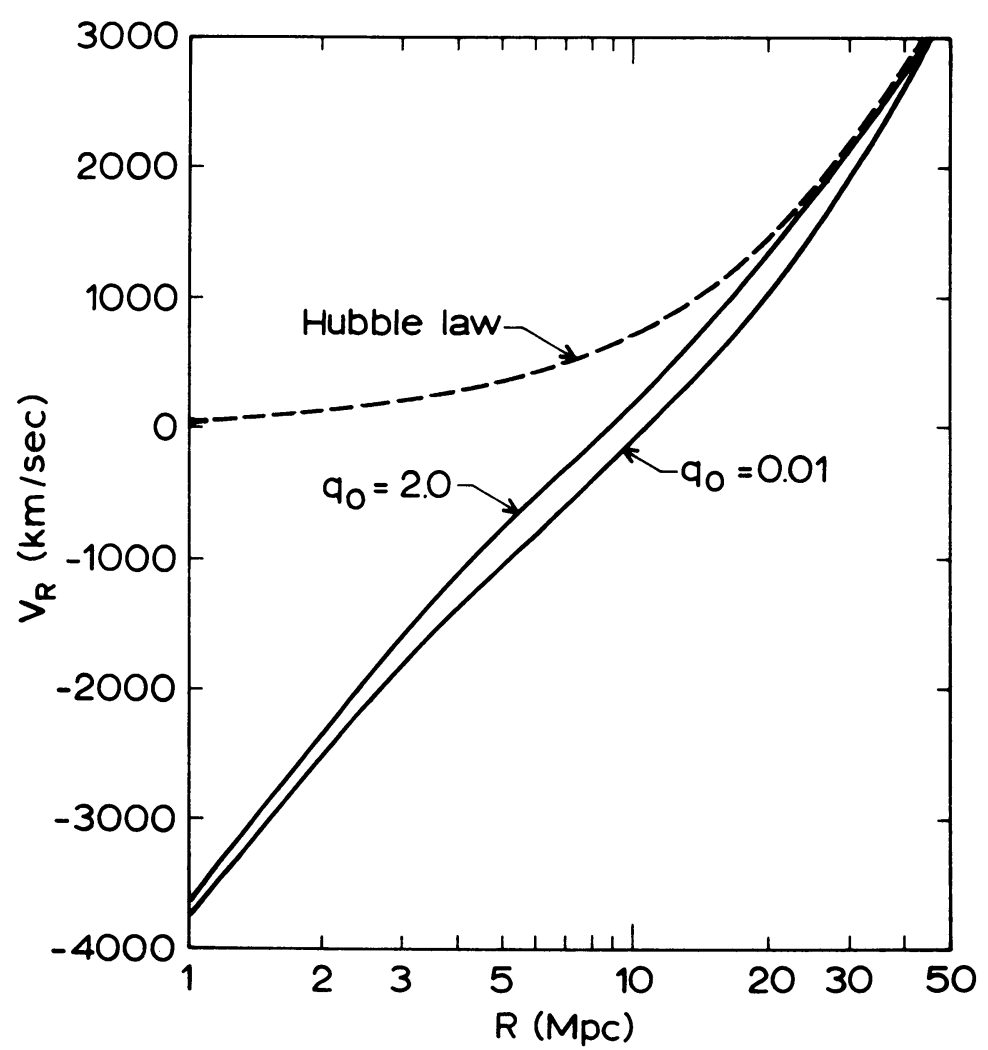

Fig. 8. Present velocity of IGG near the Coma cluster according to Gunn and Gott (1972). Note the reversal from expansion to infall at about $10 \mathrm{Mpc}$. 


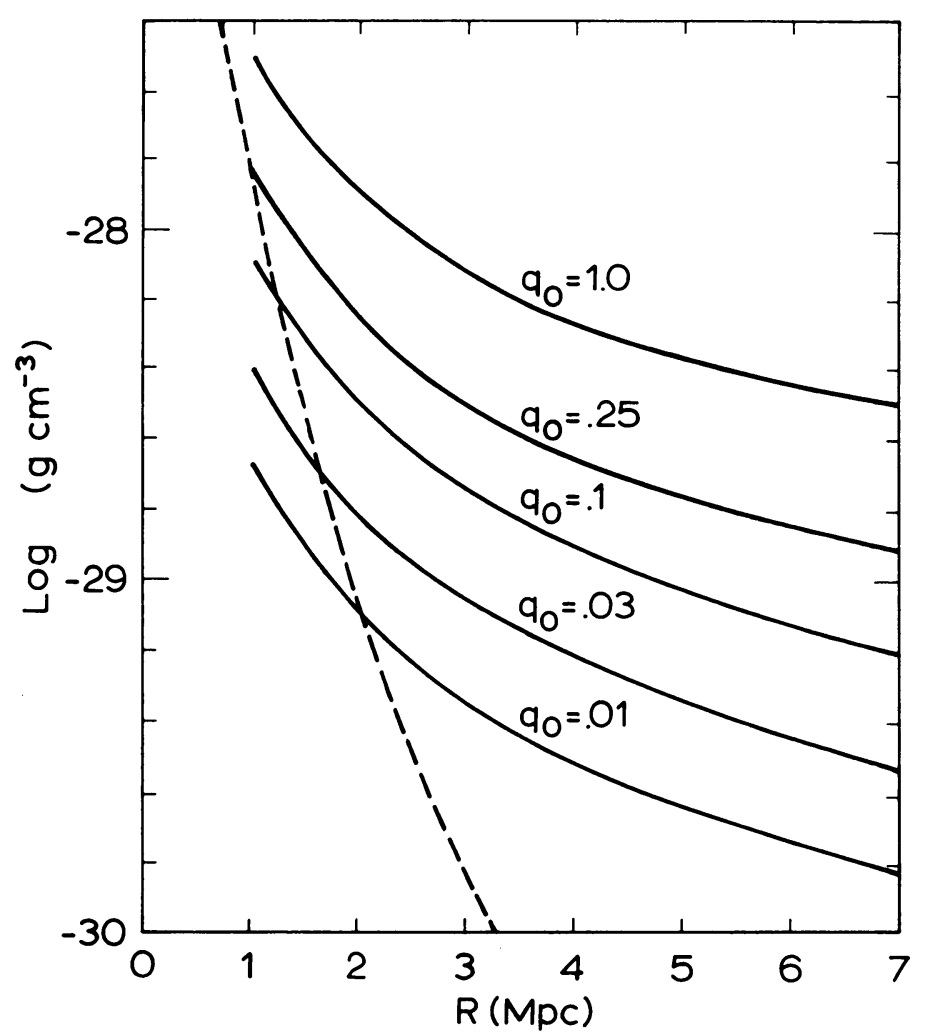

Fig. 9. Present density distribution of IGG near the Coma cluster according to Gunn and Gott (1972). The results, given for various values of $q_{0}=\Omega / 2$, show how the velocities of Figure 8 have compressed the gas above the true IGG value at $R \rightarrow \infty$.

So far, we have spoken of the diffuse X-ray background and of the discrete sources which are identified with clusters of galaxies. What is the relation between the two? In a recent paper, Silk and Tarter (1973) attempt a synthesis. They first estimate the number of clusters per unit volume for clusters of various masses, from de Vaucouleurs' groups of galaxies to Abell's richness-class $\mathrm{V}$ clusters. They also assign a probable three-dimensional velocity dispersion $\left(<100 \mathrm{~km} \mathrm{~s}^{-1}\right.$ to $\left.>3000 \mathrm{~km} \mathrm{~s}^{-1}\right)$ to each class. They then calculate the TB emission by ICG in such clusters, using the assumptions (i) that $T=\mu V^{2} / 3 R$ and (ii) that the mass of gas $M_{G}=f M_{V T}$ (virial mass). Assumption (i) is consistent with the X-ray data on Coma and assumption (ii) fits Coma if $f=3-8 \%$; the Solinger-Tucker correlation suggests that $f$ is about the same for other rich Abell clusters. Figure 10 shows the contributions of clusters to the diffuse $\mathrm{X}$-ray background as predicted by Silk and Tarter from their preferred model $a=0$ (which corresponds to assuming that all clusters have the same radius, independent of richness). The values of $f$ were chosen as follows: (i) $f=1$ for de Vaucouleurs' groups, in the absence of further information, (ii) $f=0.5$ for richness-class I from X-ray observations of Virgo, (iii) $f=0.1$ for class II from X-ray observations of 5 such clusters, and (iv) $f=0.02$ for 

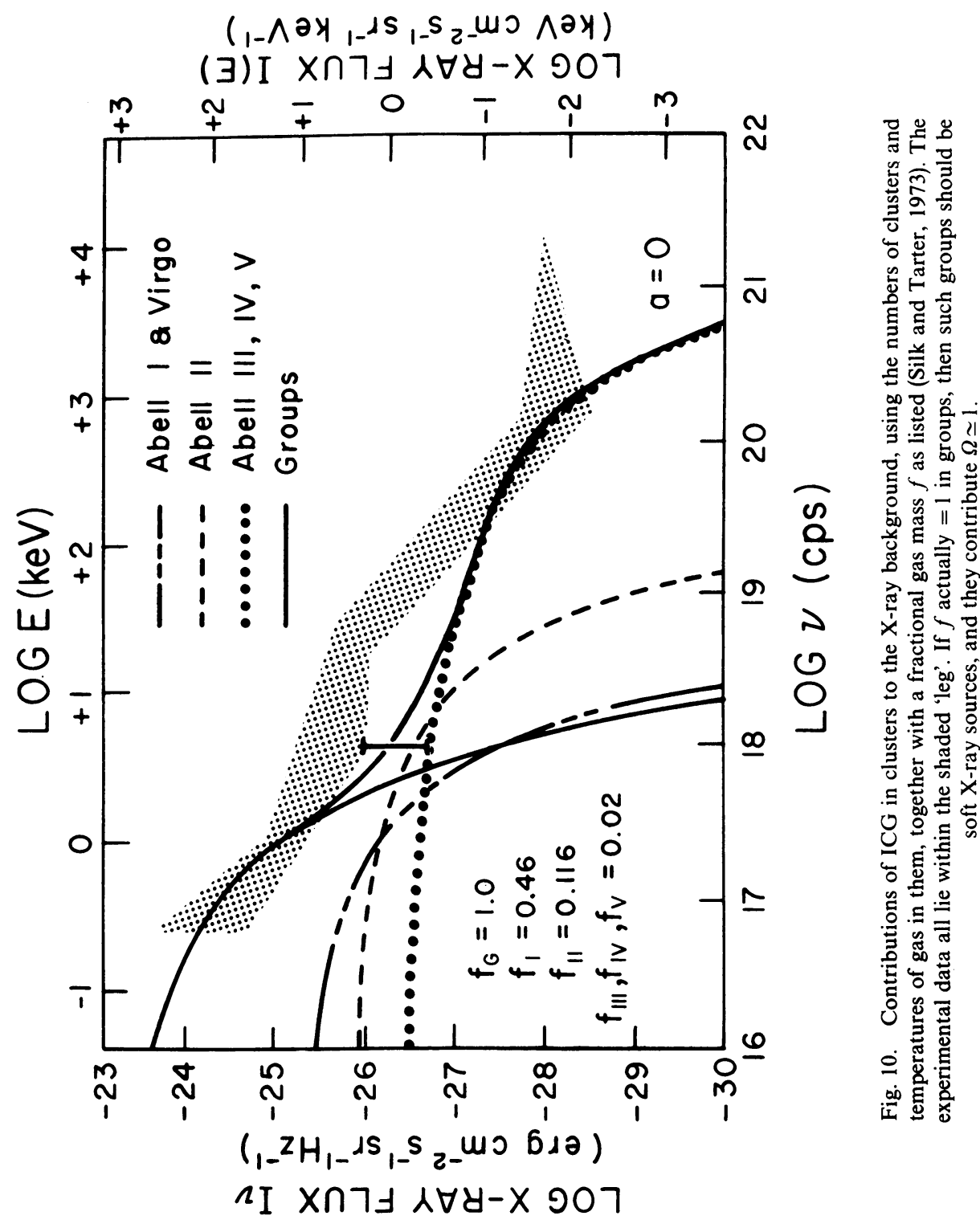
classes III to V, only to avoid the lack of isotropy in the background that would be observed if these objects were strong X-ray sources.

One sees that a substantial fraction of the observed background might be due to clusters of galaxies, ranging from $100 \%$ at $1 \mathrm{keV}$, through $10 \%$ at $10 \mathrm{keV}$, to $100 \%$ at $100 \mathrm{keV}$ and $1 \mathrm{MeV}$. It is possible that with somewhat different adopted velocity dispersions and temperatures, the $30-\mathrm{keV}$ break in the background spectrum (see Figure 1) could be explained as TB with $T_{8} \sim 3$, as suggested by Cowsik and Kobetich (1972). It is interesting that according to Silk (1973), a significant fraction of the 2-8-keV background flux can be ascribed to discrete objects of various types on the basis of actual sources observed by UHURU: (i) normal galaxies, 9\%, (ii) Seyfert galaxies, 9\%, (iii) richness-class II clusters, $9 \%$, and (iv) miscellaneous, $8 \%$, for a total of $35 \%$. Since the numbers of sources in each group involved are small (1-3) this result is very uncertain, as indicated by $\sim$ factor 3 lower estimates by Schwartz and Gursky (1973), but it is suggestive that the fraction seems to be of order unity.

What does this model say about $\Omega$ ? First of all, since rich clusters have $f<1$ and since they themselves include only a fraction of all galaxies, one would not expect their contribution to $\Omega$ to be large. In fact, Silk and Tarter find $\Omega=0.003$ for such systems. However, de Vaucouleurs' groups both are numerous and have large virial discrepancies, so it is not surprising that Silk and Tarter find $\Omega \simeq 1$ for such systems. The value of $C$ is about 7 for such groups; this roughly corresponds to the factor by which the soft X-ray observations exceed the $\Omega^{2} C=1$ line in Figure 1. Silk and Tarter therefore tentatively conclude that:

(i) de Vaucouleurs' groups of galaxies should be TB X-ray sources with $T=10^{5}$ to $10^{7} \mathrm{~K}$,

(ii) the virial discrepancy in these systems, which averages about 20 , is explained entirely by intracluster gas, and

(iii) the intracluster gas which binds the small groups is enough to close the Universe.

If these conclusions are confirmed, we will have made an important step forward in cosmology.

\section{Conclusions}

The contribution to $\Omega$ by $\mathrm{HI}$ and $\mathrm{H}_{2}$ is negligible. Ionized gas is still an open possibility. Observations of the diffuse X-ray background are sensitive to gas with $T=10^{6}$ to $10^{10} \mathrm{~K}$. From such observations, I conclude that:

(i) $\Omega^{2} C<1$ for $T>3 \times 10^{8} \mathrm{~K}$, so such gas cannot close the Universe,

(ii) $\Omega^{2} C \leqslant 1$ for $10^{7}<T<3 \times 10^{8} \mathrm{~K}$, so such gas could close the Universe if it is smoothly distributed, and

(iii) $\Omega^{2} C \leqslant 3$ for $10^{6}<T<10^{7} \mathrm{~K}$, so such gas could close the Universe even if mildly clumped.

Rich clusters of galaxies are X-ray sources. The study of such clusters, particularly Coma, suggests hot intracluster gas with $T \simeq 10^{8} \mathrm{~K}$ but the amounts are too small to bind the cluster. Attempts have been made to bind the Coma cluster with clouds having $T \simeq 10^{5} \mathrm{~K}$. This is ruled out by a combination of observations. Presumably 
such clouds therefore contribute negligibly to $\Omega$. Theoretical analysis of the accretion of intergalactic gas by rich clusters suggests $\Omega \leqslant 0.05$, but further study is required before this is accepted as final.

If intracluster gas is present in de Vaucouleurs' groups of galaxies, it may have temperatures between $10^{5}$ and $10^{7} \mathrm{~K}$ and hence radiate soft $\mathrm{X}$-rays. If the observed virial discrepancy in these groups is accounted for by gas, $\Omega=1$ and $C=7$, so $\Omega^{2} C=7$. Radiation from such gas would not violate seriously the present limit from soft $\mathrm{X}$-ray observations, $\Omega^{2} C \leqslant 3$.

I conclude that intracluster gas in de Vaucouleurs' groups may contribute $\Omega=1$, and that a decisive test would be the observation of soft X-rays from such groups.

\section{References}

Arons, J. and Wingert, D. W.: 1972, Astrophys. J. 177, 1.

Bohlin, R. C., Henry, R. C., and Swandic, J. R.: 1973, Astrophys. J. 182, 1.

Brown, R. L.: 1973, Astrophys. J. 180, L49.

Cowsik, R. and Kobetich, E. J.: 1972, Astrophys. J. 177, 585.

Davidsen, A., Bowyer, C. S., and Welch, W.: 1973, Astrophys. J. 186, L119.

De Young, D. S. : 1972, Astrophys. J. 173, L7.

De Young, D. S. and Roberts, M. S.: 1974, Astrophys. J., to be published.

Field, G. B.: 1972, Ann. Rev. Astron. Astrophys. 10, 227.

Field, G. B. and Henry, R. C. : 1964, Astrophys. J. 140, 1002.

Goldsmith, D. and Silk, J.: 1972, Astrophys. J. 172, 563.

Gorenstein, P., Bjorkholm, P., Harris, B., and Harnden, F., Jr.: 1973, Astrophys. J. 183, L57.

Gunn, J. E. and Gott, J. R.: 1972, Astrophys. J. 176, 1.

Gursky, H.: 1973, Publ. Astron. Soc. Pacific 85, 493.

Gursky, H., Kellogg, E., Murray, S., Leong, C., Tananbaum, H., and Giacconi, R. : 1971, Astrophys. J. 167. L81.

Holberg, J., Bowyer, S., and Lampton, M.: 1973, Astrophys. J. 180, L55.

Kellogg, E. M., Murray, S., Giacconi, R., Tananbaum, H., and Gursky, H.: 1973, Astrophys. J. 185 , L13.

King, I. R.: 1972, Astrophys. J. 174, L123.

Lea, S. M., Silk, J., Kellogg, E., and Murray, S.: 1973, Astrophys. J. 184, L105.

McKee, C. F. and Petrosian, V.: 1974, Astrophys. J. 189, 17.

Meekins, J. F., Gilbert, F., Chubb, T. A., Friedman, H., and Henry, R. C.: 1971, Nature 231, 107.

Miley, G. K., Perola, G. C., Van der Kruit, P. C., and Van der Laan, H. : 1972, Nature 237, 269.

Pariiskii, Yu. N.: 1972, Astron. Zh. 49, 1322. (English translation in Soviet Astron. AJ 16, 1048.)

Purcell, E. M.: 1973, private communication.

Rood, H. J., Page, T. L., Kinter, E. C., and King, I. R.: 1972, Astrophys. J. 175, 627.

Schwartz, D. and Gursky, H.: 1973, Invited papers, NASA Intl. Symposium and Workshop on Gamma-

Ray Astrophysics, to be published.

Silk, J.: 1973, Ann. Rev. Astron. Astrophys. 11, 269.

Silk, J. and Tarter, J.: 1973, Astrophys. J. 183, 387.

Smart, N.: 1973, Astron. Astrophys. 24, 171.

Solinger, A. B. and Tucker, W. H.: 1972, Astrophys. J. 175, L107.

Sunyaev, R. A. and Zel'dovich, Ya. B.: 1972, Comments Astrophys. Space Phys. 4, 173.

Welch, G. A. and Sastry, G. N.: 1971, Astrophys. J. 169, L3; 1972a, ibid. 171, L81; 1972b, ibid. 175, 323.

Wellington, K. J., Miley, G. K., and Van der Laan, H.: 1973, Nature 244, 502.

Willson, M. A. G.: 1970, Monthly Notices Roy. Astron. Soc. 151, 1.

Yahil, A. and Ostriker, J. P.: 1973, Astrophys. J. 185, 787.

Zel'dovich, Ya. B. and Sunyaev, R. A.: 1969, Zh. Eksp. Teor. Fiz. 56, 2078. (English translation in Soviet Phys. JETP 29, 1118 (1970).) 


\section{DISCUSSION}

Baum: The optical radiation from the intergalactic regions in the Coma cluster may not have anything to do with intergalactic gas. I have measured this radiation photoelectrically in two other clusters (Abell 1132 and 801) and find that it can be adequately accounted for by subthreshold dwarf galaxies plus the ordinary outskirts of the bright galaxies in these clusters. These observations do not seem to leave much margin for ascribing optical radiation to intergalactic gas, but it admittedly cannot be completely ruled out. (Reference: Baum, W. A.: 1973, Publ. Astron. Soc. Pacific 85, 530).

Field: I am very interested in what you say. Although I did not explain it in my lecture, the observations of light between the galaxies in Coma were interpreted only in terms of an upper limit, since I was aware that stellar light might contribute. Even as an upper limit, however, the optical results suffice to rule out gas of intermediate temperatures $\left(T \sim 10^{5} \mathrm{~K}\right)$ which are not ruled out by other observations, thus eliminating the possibility that Coma is bound by gas.

Rees: The calculations of the thermal X-ray background which you have described are sensitive to the assumed value of the Hubble constant, and to possible evolutionary effects. Since both these factors are uncertain, do you really believe that the apparently gratifying agreement between the observed background and the predictions for $\Omega=1$ can be more than fortuitous?

Field: There are two different points in my paper. The first is that $\Omega=1$ cannot be ruled out by the present observations. I think that this could have turned out differently. Suppose, for example, that the theory with $z_{\max }=$ some reasonable low value $(\simeq 1)$ and $C=1$ gave a value of $I_{v}$ (theor) $\gg I_{v}($ obs $)$. Then we would be safe in concluding $\Omega \ll 1$. The first point of my paper is that there is not a large difference between $I_{v}$ (theor) and $I_{v}$ (obs), so that we cannot draw this conclusion. Until the discovery of cluster X-ray sources, that is all that one could say.

The second point is that a number of clusters are X-ray sources and further, in at least one case, Coma, the distribution and spectrum of the $\mathrm{X}$-ray emission is what one expects from thermal emission by intracluster gas. It follows that at least some of the X-ray background is due to hot gas between the galaxies. According to Silk and Tarter, at some photon energies a significant fraction of the background can be explained this way. In this sense, the agreement of theory and observation is not fortuitous.

However, you are correct that it has not been proved that most or all of the background is due to IGG, so we cannot put interesting lower limits on $\Omega$. This problem remains for the future. It can be solved by $\mathrm{X}$-ray observations at the appropriate energies.

Zel'dovich: I will describe further consequences of the hot gas in the Coma cluster in the contribution of Sunyaev and myself. Compton scattering of the microwave background by these hot electrons should cause a dip in the relic radiation in the direction of the Coma cluster. Parijskij has reported the observation of such a feature. (See the paper by Sunyaev in this volume, p. 167).

Bertola: I would like to point out that the peculiar optical feature in the center of the Coma cluster is mainly due to the overlapping light distribution of single galaxies, according to counts and tracings of several deep plates.

Field: This is very interesting. Since I used the optical observations only to establish upper limits on the gas, your results would serve to depress the upper limit further and, therefore, to rule out gas as a significant contributor to the virial mass with even greater force.

Jaffe: The analysis of radio observations of the tailed source in Coma yields a value of the magnetic field in the tail and, assuming pressure balance, the pressure of the intracluster material. This is entirely consistent with the X-ray data. These tails may provide a way of investigating small clusters.

Field: Since many clusters have now been identified as X-ray sources, the first step is to observe radio galaxies in such clusters wherever they exist, and verify the relation you refer to. Then it would be interesting to go on to sources which occur in small clusters.

Another question which arises is the De Young effect I alluded to. If, as Longair argues (see below), this effect is not real, then we must explain why the gas pressure in clusters is high enough to explain 'tails', but not high enough to cause any differences between extended sources within clusters and those without.

Longair: Hooley (Monthly Notices Roy. Astron. Soc. 166, 259 (1974)) has repeated the analysis of the differences of the sizes of radio sources inside and outside clusters. Rather than use interferometric data, he has used a complete sample of radio sources for which full synthesis maps are available. He finds no difference in the mean size of double or complex sources inside and outside clusters, contrary to the claim discussed by Dr Field. It should be noted that despite the large size of the initial sample of sources, the 
final comparison is based upon rather small statistics because of the care which has to be taken to derive homogeneous samples.

Field: This is important new information, which I find perplexing. To be sure, your data are more complete than those used by De Young, but I don't see why the new information should affect the cluster sources differently from the non-cluster sources.

It is remarkable that a density of $10^{-27} \mathrm{~g} \mathrm{~cm}^{-3}$ does not appear to affect extended radio sources, since the theory predicts that even $10^{-29}$ should do so. (Of course this estimate of intracluster density depends upon the assumption that Coma and the other X-ray clusters are typical.) It would seem to follow that if your data are correct, any reasonable intergalactic gas density $\left(<10^{-29}\right)$ cannot affect extended sources either, and therefore, that ram confinement is not relevant to observed extended radio sources, either in or out of clusters. 